\title{
Jean Genet devant le miroir de la destruction de l'identité
}

\author{
Jean Genet in Front of the Mirror of Identity Destruction
}

\author{
Malak Ben Hamou \\ Université Ibn Tofail, Maroc
}

Résumé : La vie et l'œuvre de l'auteur français Jean Genet ont toujours été une quête de l'œuvre d'art absolve. Dans cette quête, trois figures majeures le guideront: le sculpteur Giacometti, le peintre Rembrandt et le funambule Abdellah Bentaga. À travers les œuvres de ces artistes, Genet découvrira l'identité absolve. Qu'est-ce qui le mena vers cette découverte et quelles conséquences eurent-elles sur l'écrivain? C'est ce rapport à l'Autre, analysé dans les quatre textes que Genet a écrit sur ces artistes, que nous nous proposons d'interroger dans cet article, notamment à travers le mythe de Narcisse.

Mołs-clés : art, altérité, identité, regard, narcissisme.

Abstract: The life and work of French author Jean Genet have always been a quest for the ultimate masterpiece. In this quest, three major figures will guide him: the sculptor Giacometti, the painter Rembrandt and the tightrope walker Abdellah Bentaga. Through the works of these artists, Genet will discover the ultimate identity. What drew him to this discovery and which consequences did it have on the writer? It is this relationship to the Other, analyzed in the four texts that Genet wrote about these artists, that we will explore in this article, particularly through the myth of Narcissus.

Keywords: art, otherness, identity, view, narcissism.

\section{Introduction}

Jean Genet s'est retrouvé dans les œuvres de Rembrandt, de Giacometti, et de Abdallah le funambule : en parlant de leur art, il n'a finalement parlé que de luimême. Leurs œuvres ont été des reflets de son propre travail d'écrivain et de l'œuvre qu'il voulait écrire. Comme Narcisse examinant son reflet dans le lac, Genet s'est examiné en scrutant leur art. S'adressant au funambule, il lui dit : " c'est Narcisse qui danse " (Genet, 2010, p. 118). Rembrandt, lui, a contemplé son reflet dans le miroir durant toute sa carrière. La figure de Narcisse, si simple en surface dans sa conception actuelle - qui est celle de l'égocentrisme -, est pourtant si complexe en profondeur. La lecture de ces quatre textes de Genet, en l'occurrence Le funambule, Le secret 
de Rembrandt, Ce qui est resté d'un Rembrandt déchiré en petits carrés bien réguliers et foutus aux chiottes, ainsi que L'atelier d'Alberto Giacometti, nous a renvoyée vers cette figure mythologique en regard de leurs quêtes intarissables de soi. Que se cache-t-il derrière l'intense contemplation de soi et vers où mène-t-elle ? C'est à ces questions que nous tenterons de répondre, notamment à travers le mythe de Narcisse.

Narcisse, figure mythologique qui donna lieu au narcissisme, - à savoir dans la psychologie moderne, l'amour excessif de soi -, est ce personnage qui tombe en admiration devant son reflet dans un lac au point d'en mourir. C'est cette version qui traverse l'esprit de tout un chacun. Et si s'observer longuement n'était finalement pas si condamnable que cela? Revenons d'abord, de manière succincte, à deux des différentes versions du mythe de Narcisse.

D'abord, celle d'Ovide dans Les Métamorphoses, qui donna naissance à l'un des mythes les plus connus de l'histoire. Fruit d'un viol, Narcisse est le fils d'un fleuve et d'une nymphe. Ce dernier hérite de la beauté de sa mère, Liriope, une beauté qui lui permettra d'être aimé de tous : «Narcisse est [donc] identifié non par son désir mais par celui de l'autre." (Aumercier, 2009, p. 85). La mère de Narcisse apprend par l'oracle Tirésias que son fils ne vivra longtemps qu'à la condition de ne jamais se connaître : "s'il ne se connaît pas » (Ovide, 1850, p. 297). Dans d'autres versions, on retrouvera plutôt s'il ne se voit pas.

Narcisse se refusera toujours à tous ses prétendants et, parmi eux, figure la nymphe Echo'. La déesse Némésis, émue par le chagrin de l'amoureuse désespérée, la prend en pitié et décide de la venger en punissant Narcisse : il souffrira lui aussi d'un amour impossible. Poussé par la soif, il se dirige un jour vers un lac et, ne se reconnaissant pas, s'éprend de son reflet. Chose curieuse alors que de définir le narcissisme comme une admiration excessive de soi puisque Narcisse ne se reconnaît_pas. II ne sait pas que cette image qui est reflétée par le lac et qui le fuit n'est autre que son propre reflet. Narcisse ne finira par se reconnaître que dans le mythe d'Ovide : «Mais c'est moi ! " avant de mourir et de se transformer en fleur.

Dans un but de rationalisation du mythe, Pausanias propose une tout autre version. Narcisse a une sœur jumelle qui meurt. Après sa mort, Narcisse contemple son reflet qui lui rappelle celui de sa sœur. Dans les deux versions, le rapport à soi et à l'autre est tout de suite posé. Devant le reflet du miroir, cet « instrument d'une universelle magie qui change les choses en spectacle, les spectacles en choses, moi en autrui et autrui en moi » (Merleau-Ponty, 1964, p. 25), est-ce moi ou un autre? Dans De pictura, Alberti désigne Narcisse comme étant l'inventeur de la peinture :

[...] j'ai l'habitude de dire à mes amis que l'inventeur de la peinture, selon la formule des poètes, a dû être ce Narcisse qui fut changé en fleur car, s'il est vrai que la peinture est la fleur de tous les arts, alors la fable de Narcisse convient parfaitement (perapta erit) à la peinture. La peinture est-elle autre chose que l'art d'embrasser ainsi la surface d'une fontaine (cit. d'après Golsenne \& Prévost, 2004, p. 101).

\footnotetext{
1 Punie par Junon, femme de Jupiter, Echo se voit privée de la parole. Elle ne pourra plus que répéter certains mots de chaque phrase qui lui est adressée.
} 


\section{La quête de soi devant son reflet}

Le mythe de Narcisse convient à l'œuvre de Rembrandt, qui se représentera une centaine de fois, jusqu'à la fin de sa vie, devant le reflet de son miroir : "C'est un phénomène unique. II n'existe dans toute l'histoire de l'art aucun exemple de ce type qui couvre une carrière artistique de plus de quarante ans. " (Midal, 2018, p. 128). Dans Le secret de Rembrandt et Ce qui est resté d'un Rembrandt..., Genet le compare à Narcisse surtout dans ses premiers tableaux. En effet, dans sa jeunesse, Rembrandt se contemple avec satisfaction devant son miroir. II se penche sur son reflet et exhibe sur ses tableaux le faste dans lequel il vit: "Dans les tableaux peints avant 1642, Rembrandt est comme amoureux du faste " (Genet, 20162). II se complait devant son image spéculaire. C'est le dur orgueil dont est accusé Narcisse aussi, la dura superbia :

Car à vingt ans le gaillard n'a pas l'air commode et il passe son temps devant la glace. Il s'aime, il se gobe, si jeune et déjà dans la glace ! Pas pour s' arranger et courir au bal, mais pour se regarder longuement, avec complaisance, en solitaire (Genet, 2013, p. 16).

L'absence d'inquiétude dans les premiers portraits est synonyme d'absence de profondeur pour Genet. Rembrandt ne cherche pas à se connaître mais à s'autosatisfaire devant son reflet à travers une "simulée quête de soi » (Genet, 2013, p. 17). La quête de soi se fait-elle nécessairement dans la douleur? C'est ce qu'avance Genet dans sa quête de l'art.

Rembrandt, dans la suffisance de ses premiers portraits, s'éloigne de la quête de soi. II est dans l'exhibition de la richesse, la vanité et la complaisance. Le premier Rembrandt est un Rembrandt jeune, arrogant, presque hypocrite : « hypocrisie des simulateurs " (Genet, 2013, p. 23). Le peintre simule cette recherche de soi alors qu'il ne fait que se contempler dans l'amour de son reflet et la fascination devant son image. Durant sa jeunesse, Rembrandt est heureux : «On sent Rembrandt heureux d'inventer ou de représenter une richesse conventionnelle, comme heureux de peindre cette extravagante Saskia en Flore [...]" (Genet, 2016). Le bonheur de Rembrandt est ressenti par l'écrivain à travers le faste qu'il représente en abondance dans ses tableaux. Le jeune peintre est satisfait de ses tableaux, de sa femme, de sa vie, de tout ce qu'il entreprend, de tout ce qu'il possède, « il est célèbre. II s'enrichit. II est fier de sa réussite. Saskia est couverte d'or et de velours... " (Genet, 2016), or le génie artistique selon Genet ne s'accomplit pas dans le bonheur et dans la légèreté, le génie, pour lui, « c'est le désespoir surmonté à force de rigueur » (cit. d'après White, 1993, p. 228).

De ce fait, le Rembrandt jeune n'est pas celui qui intéresse Genet mais plutô† le Rembrandt vieux. Néanmoins, le passage du premier Rembrandt au dernier est nécessaire dans la compréhension de son art. Ce passage n'est pas sans rappeler celui qu'évoque Genet dans son interview avec Hubert Fichte lorsqu'il parle de luimême, en l'occurrence, " des rituels de passage. De passage de la puberté à l'âge d'homme " (Genet, 1991, p. 115). Rembrandt aussi a accompli ce passage : d'un jeune homme arrogant, plein de fastes, à un autre, celui qui se faufile petit à petit, au fil de la vie et des tableaux. Rembrandt aurait-il intéressé Genet si ce passage ne s'était pas opéré ? II est évident que non. D'ailleurs, dès le début du texte, Genet le souligne : «Quand il se pose sur un tableau de Rembrandt (sur ceux de la fin de sa

2 Les citations extraites de ce texte ne sont pas paginées. 
vie) notre regard se fait lourd, un peu bovin. " (Genet, 2013, p. 11). Que s'est-il passé durant ce passage, qui a transformé Rembrandt et avec lui, son art?

Ce qui s'est passé, c'est la mort de sa femme Saskia. En effet, Genet divise l'œuvre de Rembrandt en deux périodes : celle d'avant 1642, soit d'avant la mort de Saskia, et celle d'après. Et c'est là le point central du texte, Le secret de Rembrandt : la vie d'un artiste et la façon dont elle déteint sur sa méthode ou l'inverse, c'est-à-dire sa méthode et la façon dont elle déteint sur sa vie. Si dans Ce qui est resté d'un Rembrandt... Genet s'attarde sur la révélation qu'il eut face au petit vieux dans le train et devant les tableaux du peintre hollandais, dans Le secret de Rembrandt, il explique les raisons du changement de technique chez le peintre. Dans les autoportraits de Rembrandt, Genet voit «l'évolution de sa méthode et l'action de cette évolution sur l'homme. Ça, ou bien l'inverse ? " (Genet, 2016)

Vie et œuvre sont intimement liées, intrinsèques l'une à l'autre. À la mort de Saskia, constatation étonnante de Genet, Rembrandt ne sait plus peindre: «ce fils de meunier qui à vingt-trois ans savait peindre, et admirablement, à trente-sept il ne saura plus. " (Genet, 2016). Non seulement, il a dû réapprendre à peindre selon l'écrivain "avec une hésitation presque gauche" (Genet, 2016), mais il n'ira plus jusqu'à la virtuosité. Genet ne s'attarde pas sur la technique du peintre, car ce n'est pas ce qui l'intéresse, mais si Rembrandt réapprend à peindre, sans «jamais se risquer à la virtuosité "1, c'est parce qu'il renoncera à certaines techniques qu'il maitrisait mieux que quiconque durant sa jeunesse, comme l'explique Fabrice Midal :

Il abandonne la justesse du tracé qui a toujours défini le grand art.

Il laisse de côté l'effusion de la couleur. On ne voit plus désormais ni de rouge, ni de bleu, ni de vert... mais un camaïeu d'ocres.

II ne s'occupe plus du rendu des matières, qu'il maîtrisait pourtant dans sa jeunesse comme personne (Midal, 2018, p. 139).

La mort de Saskia et le rôle primordial que lui donne Genet dans l'analyse de l'œuvre de Rembrandt, n'est pas non plus sans rappeler son article sur Les frères Karamazov de Dostoïevski en 1981, vers la fin de sa vie. Ce livre est pour Genet un chef-d'œuvre, le meilleur que l'écrivain russe ait écrit. Ce court article de trois pages et demie est un condensé de la réflexion de Genet sur le génie artistique. C'est d'ailleurs le seul texte dans lequel Genet fait la critique d'un livre : « [...] seul essai de critique littéraire que nous possédions de Genet. Une lecture des Frères Karamazov. ॥ (Neutres, 2013, p. 68). II ne nous semble pas anodin que le meilleur roman de Dostoïevski pour Genet soit ce livre en particulier, puisque durant l'écriture de ce dernier livre, un évènement tragique eut lieu dans la vie de l'écrivain russe : la mort de son fils. Dostoïevski dut-il réapprendre à écrire lui aussi après la mort de son fils ?

À la mort de Saskia, Rembrandt commence à voir autre chose devant le miroir. Le peintre se penche désormais sur une blessure, le temps de la légèreté est, semble-t-il, révolu. Dans l'un de ses autoportraits après la disparition de son épouse, Rembrandt se peint comme il avait peint auparavant sa femme dans l'un de ses portraits : «En 1643, il peint un autre portrait de lui-même en pendant de celui de Saskia. Or elle est morte le 14 juin 1642. " (Bonafoux, 19903) Comme le Narcisse du mythe de Pausanias

3 https://www.clio.fr/bibliotheque/pdf/pdf_lenigme_de_rembrandt.pdf [06/03/2021]. 
qui se penche à la recherche vaine du reflet de sa sœur disparve, - « quand la jeune fille mourut, il se rendait à la fontaine, sachant que c'était son reflet qu'il voyait mais trouvant, malgré ce savoir, quelque consolation à son amour en s'imaginant qu'il voyait non pas son reflet, mais l'apparence de sa sour " (Pausanias, 1935, pp. 310311) -, Rembrandt s'est peut-être mis à la recherche de sa défunte femme dans le miroir. Les deux versions du mythe nous semblent convenir à Rembrandt. Qu'il se penche sur le reflet de l'être aimé disparu ou sur son propre reflet, il s'agit toujours d'une recherche de soi qui relève du narcissisme.

Si la complaisance devant le miroir durant la jeunesse du peintre est narcissique pour Genet, la recherche de ce vieux Rembrandt qui n'a jamais cessé de se regarder dans sa glace n'en est pas moins narcissique aussi. En effet, toute cette quête continue et ininterrompue nous semble relever du narcissisme. Rembrandt est à la poursuite de cette image qui semble lui échapper de plus en plus au fil des années. Et même s'il se regarde avec complaisance dès sa jeunesse, Rembrandt n'en reste pas moins "un homme inquiet, à la poursuite d'une vérité qui le fuit » (Genet, 2016). ॥ s'agit de poursuivre son image devant un miroir, chez un voyageur dans un train, sur un fil, dans un tableau, une statue, ou un texte.

Devant l'u onde trompeuse » (Ovide, 1850, p. 298), le peintre regarde ce reflet qui, compte tenu de son œuvre et de sa recherche incessante devant le miroir, le fuira jusqu'à la fin de ses jours. S'il avait élucidé le secret du fantôme du miroir, il aurait sans doute arrêté d'interroger son reflet, mais Rembrandt n'arrêtera jamais. II s'obstine à courir derrière ce fantôme, comme Narcisse: «Trop crédule Narcisse, pourquoi t'obstiner à poursuivre un fantôme qui te fuit ? ॥ (Ovide, 1850, p. 299). Le narcissisme est donc la quête de soi. Comme l'affirme Fabrice Midal, le narcissisme « implique non pas une sorte de fascination stupide et aveugle pour soi mais l'exploration de l'énigme de notre propre existence" (Midal, 2018). Partant de cette définition, Rembrandt, devant son reflet, se cherche, questionne son identité, court derrière une image si proche et si lointaine à la fois. Une image qui ne cesse de fuir et de lui échapper : «Dans le mythe de Narcisse, le reflet est une identité, à la fois confirmée par la reconnaissance et contestée par une image flottante » (Malgorn, 1988, p. 60).

\section{La destruction de l'altérité à travers le regard}

Le passage de la vie à l'art est ce qui caractérise la forme du texte Ce qui est resté d'un Rembrandt... Genet passe d'un évènement de sa vie dans l'une des colonnes du texte à l'œuvre de Rembrandt dans l'autre. Dans la colonne de gauche, écrite en caractères pleins, Genet raconte sa rencontre avec un vieux dans un train. Dans celle de droite, écrite en italique, il analyse l'œuvre de Rembrandt. Ce qui relie le texte de droite à celui de gauche est la révélation qu'eut Genet : « Chacun est l'autre et les autres " (Genet, 2013, p. 19). Cet évènement que Genet vécut avec désolation, dégoût et tristesse nous semble déroutant. L'écrivain n'engagea aucune conversation avec le voyageur, ne lui adressa même pas la parole. Tout eut lieu dans le regard et dans la confrontation avec le regard de l'autre. Par cette opération "magique de la vision », Genet vit au-delà de l'apparence physique du petit vieux, qu'il trouva, par ailleurs, dégoûtante et sale : " corps et visage sans grâce, laids, selon certains détails, ignobles même [...] ॥ (Genet, 2013, p. 12).

Contrairement à Sartre, pour qui le regard de l'autre permet de se reconnaître en tant qu'individu différent, et contrairement à Merleau-Ponty pour qui le miroir permet à l'enfant de connaître l'image qu'il renvoie aux autres et donc de créer sa propre identité et de se différencier de l'autre, Genet conçoit l'identité autrement. L'autre, 
en l'occurrence le voyageur, a permis à Genet de détruire l'altérité : « Au monde il existe et il n'exista jamais qu'un seul homme. Il est tout entier en chacun de nous, donc il est nous-même. Chacun est l'autre et les autres. " (Genet, 2013, p. 19). Dans le regard du voyageur, Genet découvre l'identité absolve :

Au confort de la formule "Moi=Moi", qui signifie l'identité au premier degré, se substitue le constat dérangeant: "Moi=Lui", expression définitive de ma condition humaine dans ce qu'elle comporte d'inexorable (Macherey, 20134).

Genet se vit dans le regard du voyageur comme l'on se verrait dans le reflet d'une glace : «Dans cet affrontement avec le miroir, il y a à la fois dualité, dédoublement et unité. C'est le même qui est deux. " (Vernant, 1990, p. 126). Devant le voyageur, il se passe exactement la même chose, soit le même qui est deux et plus encore, c'est le même qui est tous. En regardant le vieux, l'altérité ne se constitue pas, contrairement à ce qu' affirme Husserl :

Admettre que c'est en moi que les autres se constituent en tant qu'autres est le seul moyen de comprendre qu'ils puissent avoir pour moi le sens et la valeur d'existences et d'existences déterminées (Husserl, 1966, p. 109).

Au contraire, l'altérité s' effrite : « [...] en regardant le voyageur assis en face de moi j'eus la révélation que tout homme en vaut un autre " (Genet, 2013, p. 11). Pour cet être unique qui est en chacun de nous, Genet utilise le mot "identité », une seule identité pour tous les hommes, avant de se corriger, comme il a l'habitude de le faire dans tous ses textes: " ce mot s'obstinait à revenir, mais peut-être parce que je ne disposais pas encore d'un très riche vocabulaire ॥ (Genet, 2013, p. 16).

Genet va encore plus loin. Cette identité, cet être fragmenté n'est pas réductible à l'homme, mais est partagé par les animaux aussi. Dans le regard d'un animal mort, Genet dit voir cette identité commune, absolve, cet être unique qui est fragmenté : «Cette équivalence je crus même la trouver, aux Halles, aux abattoirs, dans l'œil fixe, mais non sans regard, des têtes de moutons coupées " (Genet, 2013, p. 17), ou encore : « Qui eussé-je assassiné si j'avais tué tel ou tel guépard qui marchait à longues foulées, souple comme un voyou d'autrefois ? " (Genet, 2013, p. 18)

Cette expérience du train, qui mena vers cette révélation, est racontée de façon laconique dans L'atelier d'Alberto Giacometti, presque expédiée, comme si cet évènement n'avait ni beaucoup d'importance ni une réelle incidence sur Genet. Dans Ce qui est resté d'un Rembrandt ... non seulement cette rencontre avec ce petit vieux est racontée en détails mais nous découvrons aussi comment l'auteur l'a vécue et quelles émotions elle a produites en lui. Cette rencontre banale, accidentelle, changea l'auteur et le hanta à tout jamais :

Genet a connu sa nuit pascalienne, anti-pascalienne si l'on préfère, qui lui a fait prendre conscience - il le relate dans Ce qui est resté d'un Rembrandt... - de son irréductible anonymat et de son impossibilité de se constituer comme sujet: le même est l'autre et l'autre est moi (Corvin, 1996, pp. 110111).

Elle le désempara, transforma sa vision du monde, et tout comme Rembrandt, son art en changea : «Puisque divers incidents de ma vie m'avaient contraint à la poésie,

\footnotetext{
4 https://philolarge.hypotheses.org/1354 [05/03/2021].
} 
il faudrait peut-être que le poète utilisât cette découverte nouvelle pour lui. ") (Genet, 2013, p. 24). Pour la décrire, il n'utilise que des substantifs péjoratifs : tristesse, pourriture, choc, désarroi, glaire ou encore dégoût. Pourquoi a-t-elle eu lieu dans ce train, avec ce petit vieux et lors de ce voyage? Qu'est-ce que cet homme avait de particulier? Rien. Il était même laid et sale :

[...] corps et visage sans grâce, laids, selon certains détails, ignobles même: moustaches sales, ce qui serait peu, mais dures, rigides, les crins plantés presque horizontalement au-dessus de la bouche minuscule, bouche gâtée, mollards qu'il envoyait entre ses genoux sur le plancher [...] (Genet, 2013, p. 12).

Derrière ce physique peu avantageux, Genet devine un homme « probablement veule, peut-être lâche ». Au-delà du visible, l'écrivain put - pour reprendre ses mots en parlant de Rembrandt - écarter les oripeaux. Il les écarta, non de façon analytique, intelligente ou réfléchie, mais de manière accidentelle.

La révélation s'imposa à lui, avec toutes les conséquences qu'elle apporterait sur sa vie et sur son travail d'écrivain, sans aucune rationalisation de sa part : « Son regard n'était pas d'un autre: c'était le mien que je rencontrais dans une glace, par inadvertance et dans la solitude et l'oubli de moi. ") (Genet, 2013, p. 13). E† c'est pour cette raison même que cette découverte ne le quitta plus. Lui, qui rejette la luminosité analyste (Genet, 1968, p. 886), fut secoué par cette idée qui vint le troubler et qui se transforma d'intuition en certitude :

[...] c'est une chose différente que connaître d'une façon analytique en somme, et saisir par une intuition soudaine. (Car bien sûr j'avais entendu dire autour de moi, et je l'avais lu, que tous les hommes se valent, et même qu'ils sont frères.) Mais m'avancer à quoi ? ॥) (Genet, 2013, pp. 18-19).

Seulement, ce n'est pas de cette équivalence-là dont parle le poète, car il ne ressent aucun sentiment de fraternité envers l'autre. L'autre n'est pas un frère, l'autre est moi : « Aucun homme n'était mon frère : chaque homme était moi-même, mais isolé, temporairement, dans son écorce particulière. " (Genet, 2013, p. 20). La seule différence entre les hommes est l'apparence physique ou l'enveloppe charnelle.

\section{La fragmentation de l'identité}

Plusieurs corps enveloppent un seul être fragmenté en plusieurs apparences: «Les hommes ne sont que la fragmentation de cet homme unique que chacun porte en soi. L'altérité n'existe pas, sinon celle des apparences et des images. ") (Redonnet, 2000, p. 130). Dans Miracle de la rose, nous retrouvons déjà ce rapport à l'identité de Genet. II dit apprendre son visage sur le visage de l'autre dans une absence totale d'altérité :

Sans qu'il le sût, je regardais son visage que je croyais être aussi le mien. J'essayais, sans y parvenir, de graver tous ses traits dans ma mémoire. Je fermais les yeux pour essayer de le reconstituer. J'apprenais sur le sien mon visage. [ ... ] (Genet, 1946, p. 444).

Devant les statues de Giacometti aussi, c'est cette absence de différence qui fascine tant Genet. Le sculpteur lui dit en le peignant un jour : "Comme vous êtes beau. " Puis il ajoute cette constatation qui l'émerveille encore plus: "Comme tout le 
monde, hein? Ni plus, ni moins." "(Genet, 20075). Tout comme Rembrandt qui ne devint " plus qu'un regard et une main » (Genet, 2016), Giacometti n'était plus qu'un regard et une main, un regard qui enlève tout ce qui entrave la vision et une main qui le rend dans son œuvre comme l'explique Pierre Macherey:

Le sens de cette réflexion paraît clair : tout le monde, et plus généralement tout être, personne ou chose, est " beau " si on sait le regarder comme il faut. Et Giacometti dispose du bon regard à cet effet: il regarde avec ses pinceaux, qui font apparaître ce qu'il voit, de la manière dont il le voit, au fur et à mesure sur la toile (Macherey, 2000).

Ces fragments nous rendent cet être - que nous sommes tous selon Genet -, méconnaissable à cause des apparences qui, elles, ne sont qu'accidentelles : « [...] un phénomène, dont je ne connais pas le nom, semble diviser à l'infini cet homme unique, le fragmente apparemment dans l'accident et dans la forme, et rend étranger à nous-même chacun des fragments. " (Genet, 2013, p. 20). Chacun n'est ce qu'il est physiquement que par accident, sauf que l'accidentel devient primordial. II nous distingue, nous différencie, nous rend identifiable. Outre cette apparence physique, rien ne nous rend singulier, différent des autres :

Chaque homme n'avait peut-être de précieux et de réel que cette singularité : "ses" moustaches, "ses" yeux, "son" pied-bot, "son" bec-delièvre? Et s'il n'avait, pour s'enorgueillir, que la taille de "son" chibre? (Genet, 2013, p. 23)

Devant les œuvres de Rembrandt et de Giacometti, Genet se voit comme il s'est vu dans le regard du voyageur. II voit dans leurs tableaux et sculptures le travail de désidentification : "les portraits de Rembrandt (après la cinquantaine) ne renvoient à personne d'identifiable." (Genet, 2013, p. 20). Aucun portrait ne renvoie vers quelqu'un en particulier, pas même les autoportraits de Rembrandt. Devant ses tableaux, Genet voit une représentation de la vie de n'importe quel individu puisqu'une « personne en vaut une autre ». À propos des autoportraits de Rembrandt, Ricœur dit :

Narcisse aime d'un amour érotique sa propre image dans les eaux. L'embrassant, il la brise. Rembrandt, au contraire, garde la distance et choisit, apparemment sans haine ni complaisance, de s'examiner (Ricoeur, 1994, p. 15).

Or, Narcisse ne reconnaît pas son image et tout le mythe repose sur cette difficulté à se reconnaître et à s'identifier : «La démence de Narcisse consiste précisément dans le fait qu'il ne se reconnaît pas [...]. ॥ (Hadot, 1976, p. 92). Dans le mythe d'Ovide, il tombe amoureux de son image qu'il pensait celle d'un autre et dans le mythe de Pausanias, il regarde son reflet en y voyant l'image de sa sœur disparue :

Ce rappel du mythe et de quelques-unes de ses interprétations fait ressortir qu'il s'agit de la constitution du sujet, dans son rapport à soi et aux autres, et de la connaissance (ou de la méconnaissance) de soi (Roch, 2002, p. 24).

Narcisse nous pousse à nous examiner, à nous questionner ainsi qu'à questionner notre rapport à l'autre, tout comme le fait l'œuvre de Rembrandt :

\footnotetext{
${ }^{5}$ Cette version n'est pas paginée.
} 
[...] et c'est pourquoi ces deux œuvres sont nos miroirs. En se peignant, ils ne nous montrent pas tant qui ils sont eux (Montaigne et Rembrandt), mais qui nous sommes, nous. Ils nous aident ainsi à nous voir dans notre plus poignante humanité ; ils nous narcissisent. Montaigne comme Rembrandt révèlent donc le sens profond de l'aventure de la rencontre de soi [...] (Midal, 2018).

L'enveloppe charnelle, tout comme dans le mythe de Narcisse, est trompeuse. Elle nous empêche même de voir cet être unique que nous sommes tous, nous rend méconnaissable ses différents fragments et, de ce fait, crée l'altérité. L'identité fragmentée est enfermée dans l'apparence de chacun que Genet qualifie de "prison" ou encore de "tombe", ce qui nous pousse à dire que l'être que nous sommes tous mourrait dans chaque corps, qui seul fait la singularité de chacun. L'identité commune se meurt dans un nombre incommensurable de corps. L'identité est ensevelie dans les accidents de l'apparence et c'est pour cette raison même qu'il est si difficile de reconnaître cet être unique. E† c'est là où Rembrandt ou encore Giacometti réussissent, selon Genet, ce que nul autre n'a pu faire à part eux, d'où la fascination du poète pour eux : ils ont su déterrer cette identité absolve enfermée dans la prison de chacun, enterrée dans la tombe de tout être, cachée derrière le visible. Une découverte troublante et déroutante qui vient anéantir toutes les anciennes certitudes de l'écrivain.

E† c'est Rembrandt qui, avant quiconque, réussit à faire voir à Genet au-delà de ces apparences, au-delà de l'accidentel, au-delà des artifices: «Rembrandt le premier me dénonça. Rembrandt ! Ce doigt sévère qui écarte et montre... " (Genet, 2013, p. 25). Rembrandt a su enlever ce qui entrave le regard, supprimer le pittoresque, déchirer les apparences trompeuses:

Dans ses autoportraits, y compris celvi inspiré directement de ses exemples, il fait complètement disparaître toute distance sociale. II ne nous met pas face à un type social d'homme, mais face à un être humain.

Pour la première fois dans l'histoire de l'art, un peintre nous montre un homme sans histoire (Midal, 2018, p. 131).

Giacometti et Rembrandt ont réussi à voir au-delà des apparences, du visible et de tous les faux-semblants. Tout comme Genet, après sa rencontre avec le vieux du train, ils ont vu l'équivalence entre tous les êtres humains, l'absence de différence et l'incapacité à se définir en tant que sujet singulier. Ce que dit Fabrice Midal à propos de Montaigne et de Rembrandt convient parfaitement à Genet et à sa vision de l'œuvre de Rembrandt et de celle de Giacometti, ainsi qu'à sa vision de l'art en général. II utilise d'ailleurs presque la même expression que Genet en parlant de Rembrandt, c'est-à-dire écarter les oripeaux :

On croit ainsi que leur œuvre vise, de manière anecdotique, à nous communiquer leurs divers sentiments.

Or, en réalité, elle témoigne d'un travail de déprise de soi, de désidentification. Rembrandt et Montaigne se dépouillent des oripeaux de la représentation de soi, du jeu social, des identités pour découvrir une dimension plus profonde et jusqu'ici oubliée. L'humanité à nu (Midal, 2018, p. 138).

Cette révélation mena Genet à ce qu'il appelle des « renoncements ॥, les mêmes renoncements auxquels dut se soumettre Rembrandt. Des renoncements au monde, à ses vanités, à l'érotisme, aux préjugés, à la superficialité de la vie, et à l'artificiel. Rembrandt jeune est vaniteux. Rembrandt vieux est bon: «Vers la fin de sa vie, 
Rembrandt devint bon. ॥ (Genet, 2016). La vanité ou la méchanceté pour Genet sont tous ces traits de caractère qui rendraient une personne identifiable et différente des autres, c'est l'attachement au monde, à la richesse, aux apparences, à l'érotisme : " tout ce que nous nommons trait de caractère, nos humeurs, nos désirs, l'érotisme et les vanités" (Genet, 2016). La bonté qu'il attribue à Rembrandt dès le début du texte n'est pas à prendre au sens de gentillesse ou d'une quelconque qualité morale, - Genet s'étant toujours éloigné de toute moralité -, mais plutôt comme sa capacité à se détacher du monde et de ses vanités. La bonté, c'est l'absence d'érotisme, de traits de caractère, d'humeurs et de désirs :

\begin{abstract}
Mais cette bonté - ou si l'on veut détachement - il ne l'avait pas recherchée pour observer une règle morale, ni religieuse (c'est dans ses seuls moments d'abandon qu'un artiste peut avoir la foi, s'il l'a jamais) ni pour gagner quelques vertus. S'il fait passer au feu ce qu'on peut nommer ses caractères, c'est afin d'avoir du monde une vision plus pure et faire par elle une œuvre plus juste. Je suppose qu'au fond il s'en foutait d'être bon ou méchant, coléreux ou patient, rapace ou généreux... (Midal, 2018).
\end{abstract}

La bonté dont parle Genet mène vers une œuvre plus juste. Le détachement du monde et de ses vanités est ce à quoi arrive Genet aussi lors de sa rencontre avec le vieux dans le train: " D'ici peu, me dis-je, rien ne comptera de ce qui eut tant de prix: les amours, les amitiés, les formes, la vanité, rien de ce qui relève de la séduction. " (Genet, 2013, p. 22). Ce détachement ou renoncement, qu'il appelle "bonté " dans le texte sur Rembrandt, prend le nom de "mort" dans le texte sur Abdallah Bentaga, Le funambule. Car si le risque de la mort est bien réel pour le funambule, c'est d'une autre mort dont parle Genet, celle du détachement des fauxsemblants: «tu devras faire entrer en toi cette insensibilité à l'égard du monde. ॥ (Genet, 1983, p. 116).

Genet demande au funambule de se défaire de l'anecdotique, du luxe et des vanités. L'état dans lequel il voudrait que le funambule soit, se rapproche de celui du petit vieux qu'il rencontra dans le train : la saleté, la puanteur et la laideur. Cet état de saleté et de désintérêt pour son apparence physique éloigne l'artiste du monde et le laisse insensible au luxe et aux apparats. Dans son livre sur Giacometti, Genet en parle à nouveau :

II reprend la marche en boitant. II me dit qu'il a été très heureux quand il a su que son opération - après un accident - le laisserait boiteux. Voilà pourquoi je vais hasarder ceci : ses statues me donnent l'impression qu'elles se réfugient, en dernier lieu, dans je ne sais quelle infirmité secrète qui leur accorde la solitude (Genet, 20076).

II s'agit pour le funambule de fuir les faux-semblants et se réfugier dans la saleté, la puanteur et même l'infirmité, pour échapper aux artifices du monde visible et pour danser, comme Narcisse, seul devant son image qui le fuit: « Ce n'était pas une putain que nous venions voir au Cirque, mais un amant solitaire à la poursuite de son image qui se sauve et s'évanouit sur un fil de fer.» (Genet, 1983, p. 118). Le funambule est un artiste sur un fil qui court derrière son image sans jamais la rattraper. Courant derrière elle, il devra se mettre à la recherche de lui-même comme le peintre ou encore le poète. II s'agit une nouvelle fois de la quête de soi. Le funambule devra

\footnotetext{
${ }^{6}$ Cette version n'est pas paginée.
} 
n'être rien. Pour approcher l'absolu artistique, il devra renoncer au monde, n'exister que dans son art, sur son fil et ne vivre que pour son art. II lui faudra renoncer à tout pour que seul l'art subsiste :

\begin{abstract}
[...] c'est surtout parce qu'il faut que celui qui doit susciter cette image admirable soit mort, ou, si l'on y tient, qu'il se traîne sur terre comme le dernier, comme le plus pitoyable des humains, j'irais même jusqu'à lui conseiller de boiter, de se couvrir de guenilles, de poux, et de puer. Que sa personne se réduise de plus en plus pour laisser scintiller, toujours plus éclatante, cette image dont je parle, qu'un mort habite. Qu'il n'existe enfin que dans son apparition (Genet, 1983, p. 113).
\end{abstract}

Dans son texte sur Rembrandt, Ce qui est resté d'un Rembrandt ..., le jeu de miroir, et donc de la fragmentation, est poussé à son paroxysme puisque le texte est scindé en deux. Deux textes en miroirs, deux textes en regard l'un de l'autre. Écriture fragmentaire dans la forme et dans le fond puisque dans ce texte fragmenté en deux colonnes, l'une sur l'expérience de Genet dans un train et l'autre sur Rembrandt, il s'agit de la fragmentation d'un seul être, une seule identité en plusieurs apparences : « Ainsi chaque personne ne m'apparaissait plus dans sa totale, dans sa magnifique individualité : fragmentaire apparence d'un seul être, elle m'écœurait davantage. " (Genet, 2013, p. 26). Le projet de livre avorté sur Rembrandt - après la mort de Abdallah -, fut publié en fragments aussi : d'abord, Le secret de Rembrandt, ensuite Ce qui est resté d'un Rembrandt déchiré en petits carrés bien réguliers et foutus aux chiottes. Le deuxième texte sur le peintre, Ce qui est resté d'un Rembrandt..., fut même déchiré en petits fragments par Genet. Eł dans ce texte, entre une colonne et une autre, un espace blanc, un vide, une transparence, tout comme cette recherche au bout de laquelle il ne trouvera que du vide : "Ce doigt sévère qui écarte les oripeaux et montre...quoi? Une infinie, une infernale transparence.l) (Genet, 2013, p. 25). Cette transparence c'est celle de l'éternelle solitude : «E† toujours dans l'infernale contrée. C'est donc cette solitude qui va nous fasciner. ॥ (Genet, 1983, p. 118). Le vide ou la transparence c'est la solitude qui est en chacun :

[...] l'auteur perçoit en lui-même un vide - qui est peut-être cette « impossibilité de se constituer comme sujet »évoquée par Corvin -, vide qu'il montre à ses lecteurs/spectateurs par le biais de l'écriture : Genet se montre donc, ou plutôt, puisqu'il ne peut se constituer comme sujet - puisque nous ne pouvons nous constituer comme sujet - il nous montre tous tels que nous sommes au fond, dans notre solitude la plus profonde, derrière les images factices (Uvsløkk, 2002, p. 37).

Et quand cette révélation vint tout anéantir sur son passage, reste pour Genet, avec la colère, la peur et l'amour, la puissance du regard. Le regard qui anéantit le monde mais qui subsiste, plus fort que tout. Le regard destructeur, révélateur et souverain :

[...] les seuls moments de ma vie que je pouvais tenir pour vrais, déchirant mon apparence et laissant à découvert...quoi ? un vide solide qui ne cessait de me perpétuer ? - je les aurai connus lors de quelques colères vraiment saintes, dans des trovilles également bénies, et dans le rayon - le premier qui allait d'un jeune homme au mien, dans notre regard échangé. Enfin dans ce regard passant du voyageur à moi. (Genet, 2013, p. 24). 


\title{
Conclusion
}

Tout ce qui a précédé la révélation qui eut lieu dans un train, Genet l'attribue à une erreur d'optique qui, avant la rencontre du train, créait l'altérité, la singularité, la différence. L'autre ou les autres, autant de « doubles spéculaires » qui créaient cette erreur d'optique. En ne se reconnaissant pas en regardant les autres, Genet pouvait encore éprouver du désir pour eux. Une fois l'altérité détruite, l'écrivain devient insensible au désir et à l'érotisme et c'est là que réside pour lui tout le drame de cette révélation, dans l'impossibilité de tout érotisme à cause de cette identité universelle :

\begin{abstract}
J'éprouvais donc un profond dégoût pour ce vers quoi j'allais, que j'ignorais et que, grâce à Dieu, je ne pourrais éviter, puis une grande tristesse à l'égard de ce que de moi, j'allais perdre. Tout se désenchantait autour de moi, tout pourrissait. L'érotisme et ses fureurs me parurent refusés, définitivement (Genet, 2013, p. 25).
\end{abstract}

Si Genet a éprouvé du désir pour les autres avant cette révélation, tout comme Narcisse qui tombe amoureux de son reflet sans se reconnaître, c'est parce qu'il ne savait pas encore que ces autres étaient lui, que derrière l'apparence de chacun se cache un fragment de l'identité commune: "l'illusion qui trompe ses yeux irrite encore ses désirs. ॥ (Ovide, 1850, pp. 298-299). L'illusion ou l'erreur d'optique dont il parle, a attisé son désir jusqu'à cette révélation douloureuse et ravageuse. Nous entendrions presque Genet dire comme Narcisse se reconnaissant enfin : « Hélas! vain objet de ma tendresse ! » (Ovide, 1850, p. 300).

\section{Bibliographie}

ALBERTI, L.B. (2004). La peinture (De pictura). Paris : Le Seuil.

Aumercier, S. (2009). Narcisse : une histoire de cas. Le coq Héron, 196, pp. 85-89.

BONAFOUX, P. (1990). L'énigme de Rembrandt.

https://www.clio.fr/bibliotheque/lenigme_de_rembrandt.asp [06/03/2021].

CORVIN, M. (1996). Le théâtre de Jean Genet. "Une apparence qui montre le vide ». Europe, 808-809, pp. 110-123.

Genet, J. (1946). Miracle de la rose. In OEuvre complètes, vol. II. Paris : Gallimard.

Genet, J. (1968). L'Étrange mot d'... In OEuvres Complètes, vol. IV. Paris : Gallimard.

Genet, J. (1991). Entretien avec Hubert Fichte. A. Dichy, L'Ennemi déclaré: textes et entretiens. Paris : Gallimard.

Genet, J. (2007). L'atelier d'Alberto Giacometti. Paris : Gallimard.

Genet, J. (2010). Le funambule. Paris : Gallimard.

GENET, J. (2013). Ce qui est resté d'un Rembrandt déchiré en petits carrés et foutu aux chiottes. Paris : Chemin de fer.

GENET, J. (2016). Rembrandt. Paris : Gallimard.

HADOT, P. (1976). Le mythe de Narcisse et son interprétation par Plotin. Narcisses. Nouvelle revue de psychanalyse, 13, pp. 81-108. 
HuSSERL, E. (1966). Méditations cartésiennes: introduction à la phénoménologie. Paris : J. Vrin.

MACHeREY, P. (2013). Le vertige de l'identité absolve. (Genet face à Giacometti et à Rembrandt). Hypothèses. https://philolarge.hypotheses.org/1354 [05/03/2021].

MALGORN, A. (1988). Jean Genet. Qui êtes-vous? Lyon : Manufacture.

Merleau-PONTY, M. (1964). L'œil et l'esprit. Paris : Gallimard.

MIDAL, F. (2018). "Narcisse pour célébrer la beauté et la culture". In Narcisse accusé non coupable. France culture. https://www.franceculture.fr/emissions/narcisseaccuse-non-coupable/narcisse-pour-celebrer-la-beaute-et-retrouver-la-culture [05/03/2021].

MIDAL, F. (2019). Narcisse n'est pas égoïste. Paris : Flammarion.

Neutres, J. (2013). Le secret de Genet. Portrait de l'artiste en alchimiste. In VAnnouvong, A. (éd.), Genet et les arts. Dijon : Les presses du réel, pp. 59-73.

OVIDE (1850). Les métamorphoses. OEuvres complètes avec la traduction en français, 3 vol. Paris : J.-J. Dubochet, Le Chevalier et Comp.

PAUSANIAS (1935). Périégèse, t. IV, livre IX. Londres, Loeb Classical Library : W.H.S. Jones.

Ricoeur, P. (1994). Lectures 3. Aux frontières de la philosophie. Paris : Le Seuil.

RoCH, A. (2002). La forme de Narcisse. In ChIANTARETTO, J.-F. (éd.), Écriture de soi et narcissisme. Paris : Érès, pp. 21-29.

UVSLøKK, G. (2002). Le théâtre de Jean Genet. Glorification destructrice de l'Image et du Reflet. Oslo : Klassisk og romansk institutt.

VERNANT, J.-P. (1990). Figures, idoles, masques. Paris : Julliard.

WHITE, E. (1993). Jean Genet. Paris : Gallimard. 